\title{
Lutetium Lu 177 DOTA-N3-CTT1403
}

National Cancer Institute

\section{Source}

National Cancer Institute. Lutetium Lu 177 DOTA-N3-CTT1403. NCI Thesaurus. Code C158044.

A radioconjucate consisting of CT T1403, a phosphoramidate-based irreversible inhibitor of human prostate-specific membrane antigen with an albumin binding moiety, connected via click chemistry to lutetium Lu 177-dodecanetetraacetic acid-azide (177LuDOTA-N3), with potential antineoplastic activity. Upon administration, lutetium Lu 177DOTA-N3-CTT1403 targets and binds to PSMA expressed on tumor cells via its CTT1403 moiety, and upon internalization, delivers cytotoxic beta radiation directly to PSMA-expressing tumor cells. PSMA, a tumor-associated antigen (TAA) and type II transmembrane protein, is expressed on the membrane of prostatic epithelial cells and overexpressed on most prostate tumor cells. The albumin binding motif extends circulation half-life thereby improving tumor cell uptake of the radioconjugate. 